\title{
Double Hydrophilic Block Copolymer Monolayer Protected Hybrid Gold Nanoparticles and Their Shell Cross-Linking
}

\author{
Shizhong Luo, ${ }^{\ddagger}$ Jian Xu, ${ }^{\dagger}$ Yanfeng Zhang, ${ }^{\dagger}$ Shiyong Liu, ${ }^{*}, \dagger$ and $\mathrm{Chi} \mathrm{Wu}^{\ddagger, \S}$ \\ Department of Polymer Science and Engineering, University of Science and Technology of China, Hefei, \\ Anhui, 230026, China, The Hefei National Laboratory for Physical Sciences at Microscale, Hefei, \\ Anhui, China, and Department of Chemistry, The Chinese University of Hong Kong, Shatin, N.T., Hong Kong
}

Received: September 3, 2005; In Final Form: September 24, 2005

\begin{abstract}
This paper describes the syntheses of core/shell gold nanoparticles stabilized with a monolayer of double hydrophilic block copolymer and their stimuli responsiveness before and after shell cross-linking. The hybrid nanoparticles consist of gold core, cross-linkable poly(2-(dimethylamino)ethyl methacrylate) (PDMA) inner shell, and poly(ethylene oxide) (PEO) corona. First, diblock copolymer PEO- $b$-PDMA was prepared via the reversible addition-fragmentation chain transfer (RAFT) technique using a PEO-based macroRAFT agent. The dithioester end group of PEO- $b$-PDMA diblock copolymer was reduced to a thiol end group. The obtained PEO- $b$-PDMA-SH was then used to prepare diblock copolymer stabilized gold nanoparticles by the "graftingto" approach. 1,2-Bis(2-iodoethoxy)ethane (BIEE) was utilized to selectively cross-link the PDMA residues in the inner shell. The stimuli responsiveness and colloidal stability of core/shell gold nanoparticles before and after shell cross-linking were characterized by laser light scattering (LLS), UV-vis transmittance, and transmission electron microscopy (TEM). At $\mathrm{pH} 9$, the average hydrodynamic radius $\left\langle R_{\mathrm{h}}\right\rangle$ of non-crosslinked hybrid gold nanoparticles starts to increase above $35^{\circ} \mathrm{C}$ due to the lower critical solution temperature (LCST) phase behavior of the PDMA blocks in the inner shell. In contrast, $\left\langle R_{\mathrm{h}}\right\rangle$ of the shell cross-linked gold nanoparticles were essentially independent of temperature. Core/shell gold nanoparticles before and after shell cross-linking exhibit reversible swelling on varying the solution $\mathrm{pH}$. Compared to non-cross-linked core/shell gold nanoparticles, shell cross-linking of the hybrid gold nanoparticles leads to permanent core/ shell nanostructures with much higher colloidal stability and physically isolates the gold core from the external environment.
\end{abstract}

\section{Introduction}

Monolayer-protected gold nanoparticles have attracted great interest during the past decade due to their potential applications in biological imaging, medical treatment, sensors, and optoelectronics. ${ }^{1-5}$ Polymer-encapsulated gold nanoparticles typically exhibit enhanced optical and electrical properties as well as stability. ${ }^{1,2}$ The monolayer ranges from small organic compounds to macromolecules and can be further functionalized in various ways. Inspired by a reliable and facile synthesis method of preparing alkanethiol-protected gold nanopartilces reported by Brust and Schiffrin, ${ }^{6,7}$ recent progress in monolayer-protected gold nanoparticles involves using thiolated polymer ligands to stabilize gold nanoparticles, which shows that the thiolated polymer ligands are more efficient than alkanethiols in preparing small gold nanoparticles and stabilizing the particles in solutions. In general, two approaches, "grafting from" ${ }^{-12}$ and "grafting to", ${ }^{13-19}$ can be utilized to prepare gold nanoparticles covalently protected with polymer monolayers. The "grafting-from" approach allows polymers to be built up at the surface of the gold naonoparticles using surface-initiated polymerization. Endfunctionalized monolayers, regarded as macroinitiators, may be used to initiate living/controlled polymerization directly on the

* To whom correspondence should be addressed: E-mail: sliu@ ustc.edu.cn.

University of Science and Technology of China.

$\doteqdot$ The Hefei National Laboratory for Physical Sciences at Microscale.

$\S$ The Chinese University of Hong Kong. surface of gold nanoparticles. The "grafting-to" approach is especially useful to prepare polymer-stabilized gold nanoparticles because the polymer ligands can be well-defined prior to being grafted if they are synthesized by living polymerization techniques. Murray and co-workers reported the synthesis of gold nanoparticles with poly(ethylene glycol) monolayer. ${ }^{13}$ Corbierre et al. reported polystyrene-functionalized gold nanoparticles by the covalent attachment of a thiol-terminated polystyrene prepared by anionic polymerization. ${ }^{14}$

Reversible addition-fragmentation transfer (RAFT) has recently emerged as a promising controlled free radical polymerization technique due to its versatility and simplicity, and the polymer is free from the contamination of metal catalysts. Most importantly, it is compatible with almost all of the conventional free radical polymerization monomers. ${ }^{20,21}$ Polymers prepared by the RAFT technique bear dithioester end groups, which can be reduced to thiol end groups when adding a reductant $\left(\mathrm{NaBH}_{4}\right.$, $\mathrm{LiBEt}_{3} \mathrm{H}$ ) to a mixed solution of $\mathrm{HAuCl}_{4}$ and the RAFT polymer; the gold nanoparticles are then passivated with the thiolated polymers produced in situ. Using this facile approach, Lowe et al. reported the synthesis of gold nanoparticles stabilized by anionic, cationic, neutral, and zwitterionic homopolymers or block copolymers. ${ }^{16}$ Thermoresponsive gold nanoparticles coated with poly $(N$-isopropylacrylamide) (PNIPAM) monolayer have been successfully synthesized by Zhu et al. ${ }^{18}$ Tenhu et al. also prepared PNIPAM monolayer protected gold nanoparticles; they further found that thermoresponsive PNIPAM brushes 
bound to gold nanoparticles exhibit two separate phase transitions. ${ }^{22,23}$

It is well-known that amphiphilic block copolymers can selfassemble into aggregates with various morphologies in water. ${ }^{24-26}$ However, due to the presence of dynamic exchange between assembled aggregates and individual unimers, the equilibrium between the aggregates and unimers is governed by a delicate balance of weak intermolecular forces. The disruption of such a balance can be easily triggered by temperature, dilution, and salt concentration. ${ }^{27-31}$ The problem of insufficient stability of various aggregates can be addressed by structural fixation. If one block bears reactive functional groups, the morphologies of the aggregates can be fixed by chemical cross-linking. ${ }^{32-34}$ In particular, shell cross-linked (SCL) micelles combine the properties of micelles, microgels, nanoparticles, and dendrimers, and various applications such as targeted drug delivery, sequestration of metabolites, and entrapment of environmental pollutants have been suggested. ${ }^{35-39}$ The first example of SCL micelles was described by Wooley and co-workers, who oligomerized pendent styrene groups by using free radical polymerization chemistry. ${ }^{32}$ Since that seminal work, the field of covalent stabilization of micelles and vesicles has attracted increasing attention in recent years. ${ }^{40-47}$

Just recently, Taton et al. reported the preparations of core/ shell gold nanoparticles via the block copolymer self-assembly approach. ${ }^{48}$ Citrate-capped gold nanoparticles were first made hydrophobic with the introduction of 1-dodecanethiol; they were then mixed with poly(syrene-block-acrylic acid) (PS-b-PAA) in dimethylformamide (DMF), which is a common solvent for both PS- $b$-PAA and 1-dodecanthiol stabilized gold nanoparticles. Upon addition of water, which is a nonsolvent for both PS- $b$ PAA and 1-dodecanthiol stabilized gold nanoparticles, the surface-templated self-assembly of PS- $b$-PAA around hydrophobically functionalized gold nanoparticles leads to hybrid core/shell gold nanoparticles. Subsequent shell cross-linking of the PAA corona can permanently fix the core/shell nanostructure with a gold core. The advantage of this novel approach is that there is no need for ligand chemistry between gold nanoparticles and block copolymers: any nanomaterials with surface hydrophobic functionalities can be encapsulated inside micellar cores. ${ }^{48}$ One main disadvantage of this approach is that it is difficult to control the shell thickness, the number of gold nanoparticles inside each micelle, and the location of nanoparticles. Tedious separation procedures are necessary to remove empty micelles if core/shell nanostructures with one gold nanoparticle per micelle are desired.

Stimuli-responsive block copolymers and structural fixation of various self-assembled aggregates have been our continual interests in the past few years. ${ }^{39-45} \mathrm{Up}$ to now, studies on stimuli-responsive polymers mainly deal with amphiphilic and double hydrophilic block copolymers (DHBCs). ${ }^{49}$ DHBCs can self-assemble and form micellar or "reverse" micellar structures in water if external conditions such as temperature, $\mathrm{pH}$, and ionic strength are finely tuned. ${ }^{49-54}$ To the best of our knowledge, there have been no reports of hybrid gold nanoparticles stabilized by a DHBC. Here we report that a double hydrophilic block copolymer with a thiol terminal group could be used to stabilize gold nanoparticles through covalent monolayer binding. The resulting core/shell gold nanoparticles possess a well-defined three-layer microstructure, and they are stimuliresponsive. Furthermore, we demonstrated that shell crosslinking of the hybrid gold nanoparticles leads to permanent core/ shell nanostructures with much higher colloidal stability and physically isolates the gold core from the external environment.
SCHEME 1: Synthesis of Shell Cross-Linked Core/Shell Gold Nanoparticles with a Gold Core, Cross-Linked PDMA Inner Shell, and PEO outer Corona

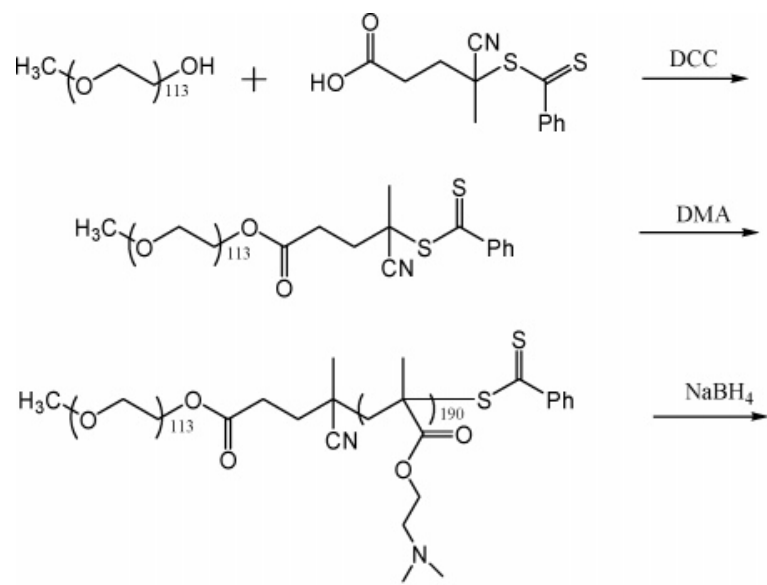

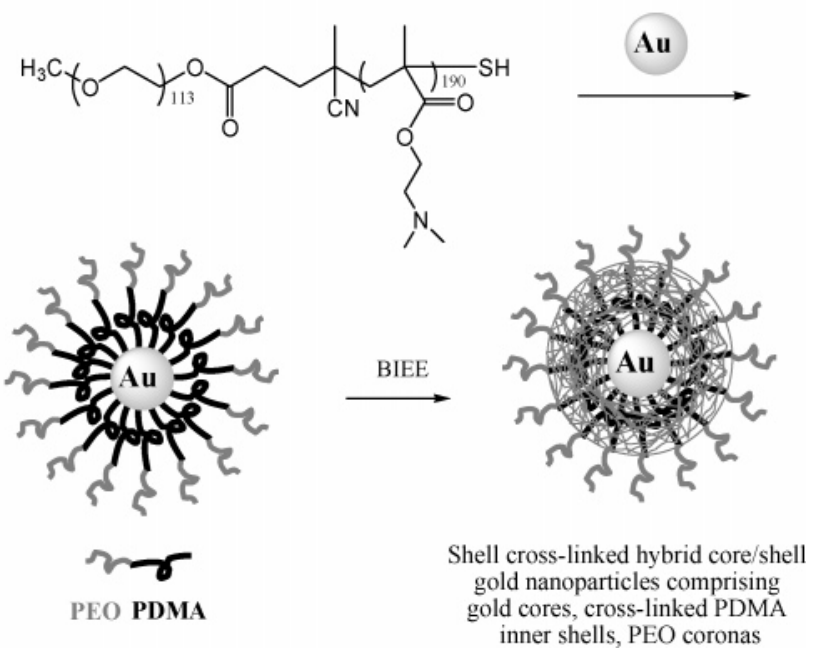

Experimental Section

Materials. 2-(Dimethylamino)ethyl methacrylate (DMA) was obtained from Aldrich and was passed through a basic alumina column, then vacuum-distilled from $\mathrm{CaH}_{2}$, and stored at $-20{ }^{\circ} \mathrm{C}$ prior to use. Monohydroxy-capped poly(ethylene oxide) (PEO-OH) with a mean degree of polymerization of $113\left(M_{\mathrm{w}} / M_{\mathrm{n}}=1.1\right)$ was purchased from Fluka. 4,4'-Azobis(4cyanopentanoic acid) (ACP) was obtained from Aldrich and was purified by recrystallization from methanol. Ethyl acetate was fraction distilled after it was dried with $\mathrm{CaH}_{2}$ overnight. 4-Cyanopentanoic acid dithiobenzoate (CPAD) was prepared according to literature procedures. ${ }^{55}$ Other regents were used as received without further purification.

Sample Preparation. A schematic illustration for the preparation of hybrid core/shell gold nanoparticles and their shell cross-linking is shown in Scheme 1.

Synthesis of Gold Nanoparticles. Gold nanoparticles were prepared via the common technique of citrate reduction, which has been described in detail in the literature. ${ }^{56}$ All glassware was rinsed with aqua regia and then several times with deionized water, and was oven-dried overnight at $160{ }^{\circ} \mathrm{C}$. Prior to use, the components of the reaction vessel were flame-dried under vacuum and then kept under dry nitrogen atmosphere. In a typical synthesis, in a $250 \mathrm{~mL}$ round-bottom flask equipped with a condenser, $100 \mathrm{~mL}$ of $1 \mathrm{mM} \mathrm{HAuCl} 4$ was brought to a rolling boil with vigorous stirring. Rapid addition of $10 \mathrm{~mL}$ of 38.8 $\mathrm{mM}$ sodium citrate to the vortex of the solution resulted in a color change from pale yellow to wine-red. Boiling was 
continued for $10 \mathrm{~min}$ and the heating mantle was removed, and stirring was continued for an additional $30 \mathrm{~min}$. After the solution reached room temperature, it was filtered through a $0.8 \mu \mathrm{m}$ Millipore membrane filter. The colloidal gold nanoparticle solution was stored at room temperature in a dark bottle.

Synthesis of PEO macroRAFT Agent. In a $250 \mathrm{~mL}$ one-neck round-bottom flask equipped with a magnetic stirring bar, PEO$\mathrm{OH}(10.0 \mathrm{~g})$ was dissolved in $150 \mathrm{~mL}$ of toluene. After azeotropic distillation of $10 \mathrm{~mL}$ of toluene at reduced pressure to remove traces of water, $0.5650 \mathrm{~g}$ of CPAD and $0.0623 \mathrm{~g}$ of 4-dimethylaminopridine (DMAP) were added. When the solution was homogenized by stirring, $1.1598 \mathrm{~g}$ of 1,3-dicyclohexylcarbodiimide (DCC) was added in portions. The reaction mixture was stirred at room temperature for 3 days. The precipitated urea was filtered. PEO-based macroRAFT agent with pink color was obtained by precipitation of the filtrate into excess of diethyl ether three times, and then dried under vacuum at room temperature for 2 days.

Synthesis of Dithioester-Terminated PEO-b-PDMA. The RAFT polymerization was conducted in a sealed glass ampule equipped with a magnetic stirring bar. In a typical run, the glass ampule was charged with $36 \mathrm{mg}$ of ACP, $4.0 \mathrm{~g}$ of DMA, $0.5 \mathrm{~g}$ of PEO macroRAFT agent, and $2.5 \mathrm{~mL}$ of ethyl acetate used as solvent. The homogenized reaction mixture was subjected to three freeze-thaw cycles to remove oxygen, flame sealed under vacuum, and placed in an oil bath at $60{ }^{\circ} \mathrm{C}$ for $24 \mathrm{~h}$ to complete the polymerization. The polymerization product was precipitated into anhydrous ethyl ether and washed with $n$-hexane several times to remove residual DMA monomer. The precipitation-washing cycle was repeated three times. After purification, the product was dried in a vacuum oven at room temperature overnight. The conversion is about $72 \%$. The degree of polymerization (DP) of PDMA block was determined to be 190 by ${ }^{1} \mathrm{H}$ NMR. The molecular weight and molecular weight distribution of $\mathrm{PEO}_{113}-b$-PDMA 190 were characterized by size exclusion chromatography: $M_{\mathrm{n}}=45300 ; M_{\mathrm{w}} / M_{\mathrm{n}}=1.15$.

Synthesis of PEO-b-PDMA Monolayer Protected Gold Nanoparticles. Thiolated PEO- $b$-PDMA was prepared by reduction of dithioester-terminated PEO- $b$-PDMA with $\mathrm{NaBH}_{4}$. To a 100 $\mathrm{mL}$ round-bottom flask equipped with a magnetic stirring bar, $0.3 \mathrm{~g}$ of dithioester-terminated PEO- $b$-PDMA was dissolved in $30 \mathrm{~mL}$ of deionized water; $875 \mu \mathrm{L}$ of $1.0 \mathrm{M} \mathrm{NaBH}_{4}$ aqueous solution was added under vigorous stirring. The reaction mixture was stirred at room temperature for more than 3 days to ensure that the dithioester terminal groups were completely reduced to yield PEO- $b$-PDMA terminated with thiol end groups, PEO$b$-PDMA-SH. For the synthesis of PEO- $b$-PDMA monolayer protected gold nanoparticles, PEO- $b$-PDMA-SH was dissolved in water at $\mathrm{pH} 5$ and at a concentration of $1.33 \times 10^{-2} \mathrm{~g} / \mathrm{mL}$; a calculated amount of citrate-capped gold nanoparticles (at a thiol/gold molar ratio of 1:100) was added dropwise under vigorous stirring. After 3 days of equilibrium at room temperature, the reaction solution was centrifuged at $15000 \mathrm{rpm}$ for $1.5 \mathrm{~h}$ at room temperature and the supernatant was removed. This process removed free polymer chains that were not conjugated to gold nanoparticles and was repeated three times. Finally, pure PEO- $b$-PDMA monolayer protected gold nanoparticles were obtained. They was redispersed in water at $\mathrm{pH} 5$ by stirring for $12 \mathrm{~h}$.

Synthesis of Shell Cross-Linked Hybrid Gold Nanopartilces. Shell cross-linking was achieved by adding 1,2-bis(2-iodoethoxy)ethane (BIEE) at $\mathrm{pH} 9$ and stirring the solution for at least 3 days at room temperature. The target degree of quaternization was $60 \%$ relative to DMA residues, allowing for the bifunctionality of BIEE. All the samples were clarified with a $0.8 \mu \mathrm{m}$ Millipore membrane filter to remove dust before characterization.

Characterization. Nuclear Magnetic Resonance (NMR) Spectroscopy. All ${ }^{1} \mathrm{H}$ NMR spectra were recorded using a Bruker $300 \mathrm{MHz}$ spectrometer. PEO-OH, CPAD, PEO macroRAFT agent, and dithioester-teriminated PEO- $b$-PDMA were analyzed in $\mathrm{CDCl}_{3}$.

Size Exclusion Chromatography (SEC). Molecular weights and molecular weight distributions were determined by SEC using a series of three linear Styragel columns HT3, HT4, and HT5 and an oven temperature of $60{ }^{\circ} \mathrm{C}$. A Waters 1515 pump and Waters 2414 differential refractive index detector (set at $30{ }^{\circ} \mathrm{C}$ ) were used. The eluent was DMF $+1 \mathrm{~g} / \mathrm{L} \mathrm{LiBr}$ at a flow rate of $1.0 \mathrm{~mL} / \mathrm{min}$.

Laser Light Scattering (LLS). A commercial spectrometer (ALV/DLS/SLS-5022F) equipped with multi- $\tau$ digital time correlation (ALV5000) and a cylindrical $22 \mathrm{~mW}$ UNIPHASE $\mathrm{He}-\mathrm{Ne}$ laser $\left(\lambda_{0}=632 \mathrm{~nm}\right)$ as the light source was used. In static LLS, we can obtain the weight-average molar mass $\left(M_{\mathrm{w}}\right)$ and the $z$-average root-mean-square radius of gyration $\left(\left\langle R_{\mathrm{g}}{ }^{2}\right\rangle^{1 / 2}\right.$ or written as $\left.\left\langle R_{\mathrm{g}}\right\rangle\right)$ of polymer chains in a dilute solution from the angular dependence of the excess absolute scattering intensity, known as the Rayleigh ratio $R_{\mathrm{vv}}(q)$, as

$$
\frac{K C}{R_{\mathrm{vv}}(q)} \approx \frac{1}{M_{\mathrm{w}}}\left(1+\frac{1}{3}\left\langle R_{\mathrm{g}}{ }^{2}\right\rangle q^{2}\right)+2 A_{2} C
$$

where $K=4 \pi n^{2}(\mathrm{~d} n / \mathrm{d} C)^{2} /\left(N_{\mathrm{A}} \lambda_{0}{ }^{4}\right)$ and $q=\left(4 \pi n / \lambda_{0}\right) \sin (\theta / 2)$ with $N_{\mathrm{A}}, \mathrm{d} n / \mathrm{d} C, n$, and $\lambda_{0}$ being the Avogadro number, the specific refractive index increment, the solvent refractive index, and the wavelength of the laser light in a vacuum, respectively; $A_{2}$ is the second virial coefficient. The specific refractive index increment was determined by a precise differential refractometer. Strictly speaking, here $R_{\mathrm{vv}}(q)$ should be $R_{\mathrm{vu}}(q)$ because there is no analyzer before the detector. However, the depolarized scattering of the solution studied is insignificant so that $R_{\mathrm{vu}}(q)$ $\sim R_{\mathrm{vv}}(q)$.

In dynamic LLS, the Laplace inversion of each measured intensity-intensity-time correlation function $G^{(2)}(q, t)$ in the self-beating mode can lead to a line-width distribution $G(\Gamma)$. For a pure diffusive relaxation, $\Gamma$ is related to the translational diffusion coefficient $D$ by $\left(\Gamma / q^{2}\right)_{C \rightarrow 0, q \rightarrow 0} \rightarrow D$, or further to the hydrodynamic radius $\left\langle R_{\mathrm{h}}\right\rangle$ via the Stokes-Einstein equation $\left\langle R_{\mathrm{h}}\right\rangle$ $=\left(k_{\mathrm{B}} T / 6 \pi \eta_{0}\right) / D$, where $k_{\mathrm{B}}, T$, and $\eta_{0}$ are the Boltzmann constant, the absolute temperature, and the solvent viscosity, respectively.

$U V-V i s$ Spectroscopy and Transmittance Measurements. The $\mathrm{UV}$-vis spectra and transmittance were acquired on a Unico UV/vis 2802PCS spectrophotometer. The transmittance of the solution was measured at a wavelength of $800 \mathrm{~nm}$ using a thermostatically controlled cuvette.

Thermogravimetric Analysis (TGA). The amount of PEO- $b$ PDMA-SH ligand on the gold core surface was determined by a PE TGA-7 instrument in a flowing nitrogen atmosphere. The temperature was increased from 20 to $800{ }^{\circ} \mathrm{C}$ at a rate of 20 ${ }^{\circ} \mathrm{C} / \mathrm{min}$.

Transmission Electron Microscopy (TEM). TEM observations were conducted on a Philips CM 120 electron microscope at an acceleration voltage of $100 \mathrm{kV}$. The sample for TEM observations was prepared by placing $10 \mu \mathrm{L}$ of hybrid gold nanoparticle solution on copper grids coated with thin films of Formvar and carbon successively. No staining was required. 


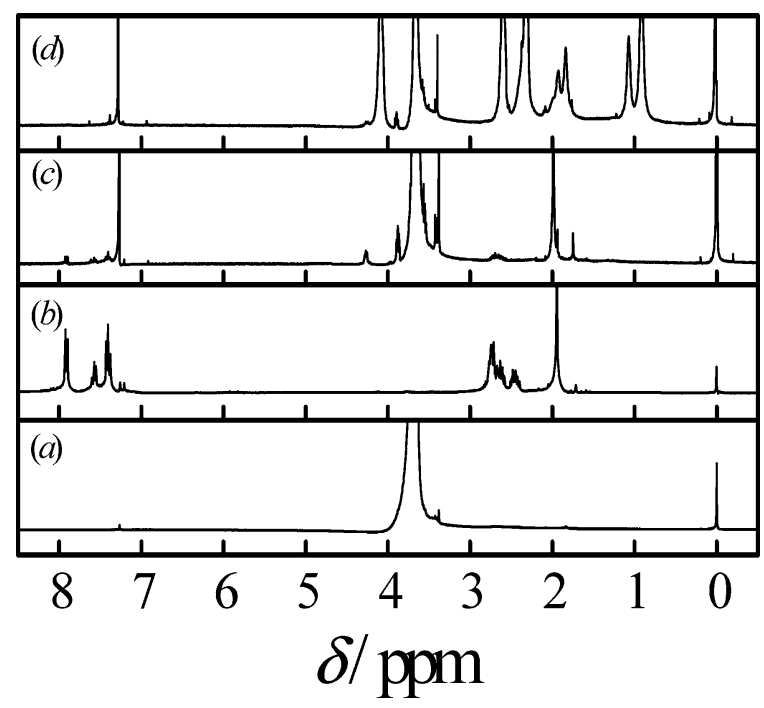

Figure 1. ${ }^{1} \mathrm{H}$ NMR spectra of (a) PEO-OH, (b) CPAD, (c) PEO macroRAFT agent, and (d) dithioester-terminated PEO- $b$-PDMA in $\mathrm{CDCl}_{3}$, respectively.

\section{Results and Discussion}

Synthesis of PEO- $\boldsymbol{b}$-PDMA-SH. A general approach to prepare shell cross-linked hybrid core/shell gold nanoparticles is shown in Scheme 1, where the small gray sphere represents the gold nanoparticle core, which is coated with a diblock copolymer monolayer.

Figure 1 shows the ${ }^{1} \mathrm{H}$ NMR spectra of PEO-OH, CPAD, PEO macroRAFT agent, and dithioester-terminated PEO- $b$ PDMA, respectively. In the spectrum of CPAD (Figure 1b), the resonances at $\delta \sim 7.3-8.0 \mathrm{ppm}$ are ascribed to the aromatic protons of the phenyl group, and signals at $\delta \sim 2.5-3.0 \mathrm{ppm}$ and $\delta \sim 2.0 \mathrm{ppm}$ represent the methylene protons and methyl protons of CPAD. Figure 1c shows the spectrum of PEO-based macroRAFT agent. Characteristic signals due to PEO and CPAD can be clearly observed. Compared to the spectra of PEO-OH and CPAD, the appearance of a new signal at $\delta \sim 4.2 \mathrm{ppm}$ is due to methylene protons of newly formed ester groups. By comparing intensity ratio of characteristic signals at $\delta \sim$ 7.3-8.0 to that at $\delta \sim 3.5$, which are ascribed to CPAD and $\mathrm{PEO}$, respectively, the degree of functionalization is calculated to be $\sim 98 \%$.

RAFT polymerization of DMA using PEO macroRAFT agent was conducted in ethyl acetate at $60{ }^{\circ} \mathrm{C}$. Rizzardo et al. have investigated the RAFT polymerization using PEO marcoRAFT agent and stated that the polymerization can be carried out in a controlled manner. ${ }^{57}$ Here we did not study in detail the kinetics of RAFT polymerization. Figure 1d shows the spectrum of dithioester-terminated PEO- $b$-PDMA, where the new signals at $\delta \sim 4.2,2.6,2.4,1.9$, and $1.0 \mathrm{ppm}$, which are characteristic of PDMA block, can be clearly observed. The degree of polymerization (DP) of PDMA block is determined by ${ }^{1} \mathrm{H}$ NMR to be 190. The molecular weight and molecular weight distribution of $\mathrm{PEO}_{113}-b$-PDMA 190 were characterized by SEC, resulting in $M_{\mathrm{n}}=45300$ and $M_{\mathrm{w}} / M_{\mathrm{n}}=1.15$. The relatively narrow polydispersity partially indicated the "living" character of the RAFT polymerization.

Figure 1d also reveals the presence of dithioester end groups after enlargement. Dithioester-terminated PEO- $b$-PDMA was reduced to PEO- $b$-PDMA-SH with the addition of $\mathrm{NaBH}_{4}$. The complete transformation of dithioester end groups into thiol groups is evidenced by the total disappearance of signals due to dithioester end groups at $\delta \sim 7.4-8.0$ ppm. ${ }^{16-18}$ SEC curves

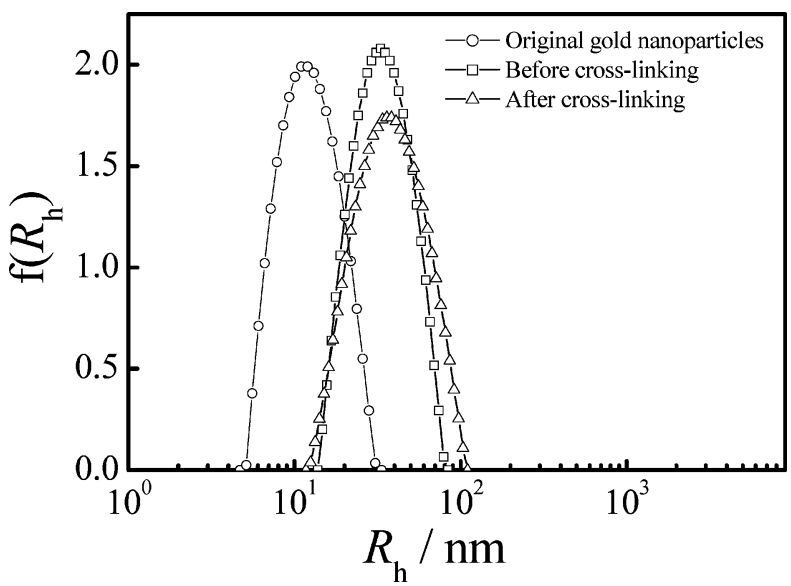

Figure 2. Comparison of hydrodynamic radius distributions, $f\left(R_{\mathrm{h}}\right)$, of original gold nanoparticles, non-cross-linked core/shell gold nanoparticles, and cross-linked core/shell gold nanoparticles at $\mathrm{pH} 9$ and 20 ${ }^{\circ} \mathrm{C}$. The scattering angle is $90^{\circ}$.

of dithioester-terminated PEO- $b$-PDMA and PEO- $b$-PDMA-SH show no appreciable differences, indicating that the reduction process results in no chain degradation.

LLS Characterization. PEO- $b$-PDMA-SH will adsorb on citrate-capped gold nanoparticles through $\mathrm{Au}-\mathrm{S}$ interaction to form a diblock monolayer. The resulting nanostructure consists of gold core, soluble PDMA inner layer, and PEO outer corona. Shell cross-linking of the hybrid core/shell gold nanoparticles was conducted upon addition of BIEE. BIEE reacts with the PDMA residues on adjacent block copolymer chains to covalently fix the three-layer nanostructure (Scheme 1). BIEE has relatively poor water solubility, and it was initially dispersed as droplets. After ca. $30 \mathrm{~min}$, these droplets disappeared and a homogeneous solution was formed; the mixed solution was stirred at $\mathrm{pH} 9$ for 3 days at room temperature. As reported previously, ${ }^{42}$ for the shell cross-linking of $\mathrm{ABC}$ triblock copolymer micelles, the actual degree of quaternization increases monotonically with the target degree of quaternization, but the former is always significantly less than the latter.

Figure 2 compares the hydrodynamic radius distributions, $f\left(R_{\mathrm{h}}\right)$, of the original citrate-capped gold nanoparticles, the noncross-linked core/shell hybrid gold nanoparticles, and the crosslinked core/shell hybrid gold nanoparticles at $20^{\circ} \mathrm{C}$. All three size distribution curves are monomodal, indicating the absence of any secondary aggregates. $R_{\mathrm{h}}$ of the original gold nanoparticles ranges from 6 to $28 \mathrm{~nm}$ with the peak located at $12 \mathrm{~nm}$. For the non-cross-linked hybrid gold nanoparticles, $R_{\mathrm{h}}$ is in the range $14-85 \mathrm{~nm}$ with the peak located at $33 \mathrm{~nm}$. The $R_{\mathrm{h}}$ of the cross-linked hybrid nanoparticles ranges from 12 to $115 \mathrm{~nm}$ with the peak located at $37 \mathrm{~nm}$. The size distribution slightly broadens and shifts to larger values after cross-linking of the PDMA inner layer. The cross-linking reaction is accompanied by quaternization of DMA units; this will introduce positive charges at the PDMA block. Charge repulsion between DMA units from the same PDMA block and from neighboring PDMA blocks, i.e., intrachain and interchain charge repulsion, in the monolayer will both cause the polymer chain to take a more extended conformation. The polydispersity indexes of the size distributions $\left(\mu_{2} / \Gamma^{2}\right)$ of original, non-cross-linked, and crosslinked hybrid gold nanoparticles are 0.07, 0.11, and 0.13, respectively. On the basis of the above results, we conclude that cross-linking reaction takes place exclusively in the PDMA inner shell surrounding the gold core, thanks to the steric hindrance exhibited by the PEO chains in the outer corona. Previously we have used the same principle to synthesize shell 


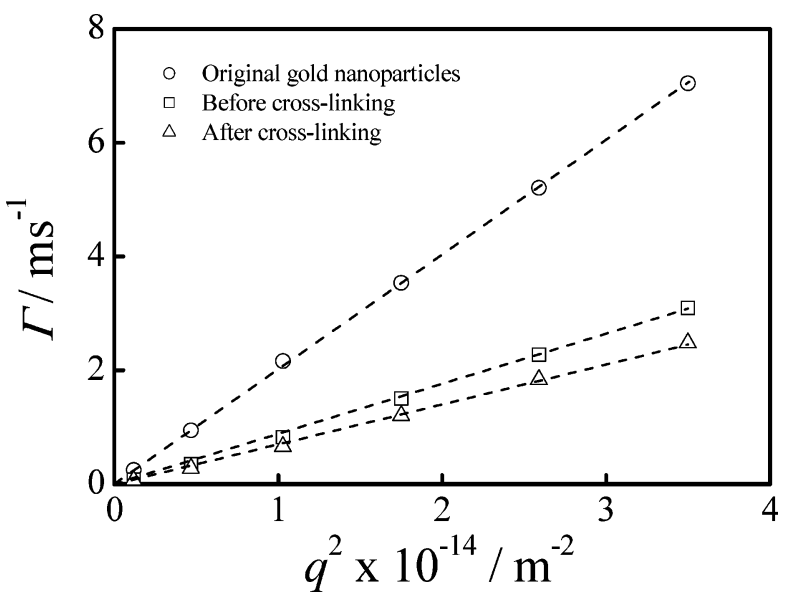

Figure 3. Scattering vector $(q)$ dependence of the average characteristic line width, $\Gamma$, of original gold nanoparticles, non-cross-linked core/ shell gold nanoparticles, and cross-linked core/shell gold nanoparticles at $\mathrm{pH} 9$ and $20^{\circ} \mathrm{C}$.

cross-linked micelles in high solids using an ABC triblock copolymer; ${ }^{42}$ here the micellar core was replaced with a gold nanoparticle.

Figure 3 shows plots of $\Gamma$ versus $q^{2}$ for original gold nanoparticles, core-shell gold nanoparticles before shell crosslinking, and core-shell gold nanoparticles after shell crosslinking,respectively. It can be seen that the average line width $\Gamma$ is a linear function of $q^{2}$ and all three lines pass through the origin, indicating that the relaxation process measured in dynamic LLS is isotropic diffusive. The slope of the line in Figure 3 leads to the average translational diffusion coefficient $D$, that is, $\Gamma / q^{2}=D$. The average hydrodynamic radius $\left(\left\langle R_{\mathrm{h}}\right\rangle\right)$ of the original gold nanoparticles is $\sim 12 \mathrm{~nm}$. The $\left\langle R_{\mathrm{h}}\right\rangle$ value of core/shell hybrid gold nanoparticles before and after shell crosslinking is 33 and $38 \mathrm{~nm}$, respectively.

TGA analysis of the non-cross-linked hybrid gold nanoparticles reveals that the organic part is about $31 \% \mathrm{w} / \mathrm{w}$. The average area at the gold core surface per diblock copolymer chain can be estimated to be $1.67 \mathrm{~nm}^{2} /$ chain, combined with the diameter of the gold core $(24 \mathrm{~nm})$ determined by dynamic LLS. Comparing $\left\langle R_{\mathrm{h}}\right\rangle$ of citrate-capped gold nanoparticles (12 $\mathrm{nm})$ and PEO- $b$-PDMA-SH monolayer protected gold nanoparticles before cross-linking $(33 \mathrm{~nm})$, the average length of a surface attached diblock copolymer chain is about $21 \mathrm{~nm}$, whereas the calculated end-to-end distance of fully extended diblock copolymer chain is $\sim 76 \mathrm{~nm}$. In preliminary experiments, we found that $\left\langle R_{\mathrm{h}}\right\rangle$ of free $\mathrm{PEO}_{113}-b$ - $\mathrm{PDMA}_{190}$ chain in water is about $5 \mathrm{~nm}$; i.e., the dimensions of free diblock copolymer chains are $\sim 10 \mathrm{~nm}$. This indicates that the surface attached polymer chains are neither highly extended nor do they adopt a random coil conformation: they exist in partially extended conformation due to the restrictions imposed by the neighboring chains in the surrounding monolayer. The dynamic LLS results agree favorably with those obtained from PNIPAM and PS stabilized gold nanoparticles, where chain extension was observed. ${ }^{19,58}$ After cross-linking, the average length of the diblock copolymer chain in the monolayer is about $26 \mathrm{~nm}$; i.e., the chains are more extended due to charge repulsion between neighboring DMA units.

Surface Plasmon Bands of Gold Nanoparticles. The optical properties of the gold nanoparticles can be tuned by varying the composition of the polymer shell, which is consistent with the theoretical prediction that surface plasmon resonance energies decrease as the refractive index of the surrounding medium increases. The position of the plasmon band is sensitive

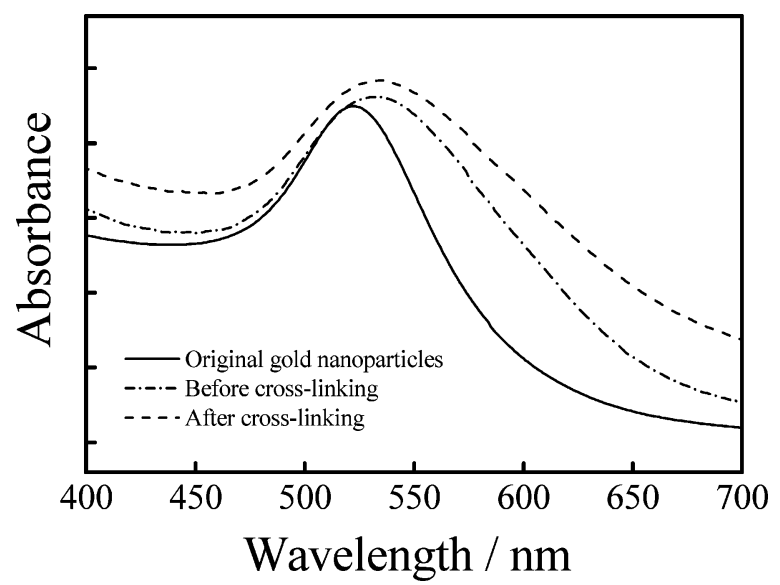

Figure 4. Absorbance spectra of original gold nanoparticles, non-crosslinked core/shell gold nanoparticles, and cross-linked core/shell gold nanoparticles in aqueous solution at $\mathrm{pH} 9$ and $20^{\circ} \mathrm{C}$.

to particle size and shape as well as to the optical and electronic properties of the particle surroundings. ${ }^{59} \mathrm{UV}$-vis spectroscopy was used to characterize the citrate-capped gold nanoparticles, the non-cross-linked core/shell gold nanoparticles, and the crosslinked core/shell gold nanoparticles (Figure 4). All three curves reveal the asymmetric band centered at $520-540 \mathrm{~nm}$, which is consistent with the presence of gold nanoparticles. Compared to non-cross-linked gold nanoparticles, the absolute absorption increases a little bit after cross-linking. This can be ascribed to increased overall scattering; i.e., the weight-average molar mass $\left(M_{\mathrm{w}}\right)$ of hybrid nanoparticles is expected to increase upon crosslinking due to the introduction of the cross-linker, BIEE. The citrate-capped gold particles have a characteristic absorbance maximum $\lambda_{\max }=523 \mathrm{~nm}$ in water, which is consistent with the diameter $(24 \mathrm{~nm})$ determined by dynamic LLS. For the core/ shell hybrid gold nanoparticles before and after cross-linking, absorbance spectra show a slight shift of $\lambda_{\max }$ to $532 \mathrm{~nm}$. The red shift in $\lambda_{\max }$ could be because PEO- $b$-PDMA monolayer has a higher refractive index compared to that of water, which will lead to a red shift in the absorbance spectra. Similar plasmon shifts have been reported for gold nanoparticles with silica shells in which the higher refractive index of $\mathrm{SiO}_{2}$ results in higher values of $\lambda_{\text {max }}{ }^{13,60}$ Taton et al. prepared core/shell gold nanoparticles by micellar encapsulation of hydrophobically modified gold nanoparticles via a self-assembly approach and reported similar behavior. ${ }^{48}$ Comparing to that of the citratecapped gold nanopartilces, the core-shell hybrid gold nanoparticles exhibit larger surface plasmon bandwidth. For the core/ shell gold nanoparticles before and after cross-linking, their absorbance maxima are at the same position $(532 \mathrm{~nm})$; this again supports that cross-linking only takes place inside the inner DMA layer and internanoparticle cross-linking is negligible.

Transmission electron microscopy (TEM) was employed to examine the core/shell gold nanoparticles after shell crosslinking. Figure 5 shows a typical TEM image of shell crosslinked hybrid nanoparticles at $\mathrm{pH}$ 9. Approximately spherical nanoparticles with a mean diameter of $18 \mathrm{~nm}$ are observed; this should be ascribed to the gold core. A closer examination of the TEM image reveals mostly isolated nanoparticles; at the same time, some hybrid nanoparticles seem to be in contact with each other, possibly due to concentration and drying effects during sample preparation for TEM. TEM observations of specimens prepared from more dilute nanoparticle solutions reveal that nanoparticles remain isolated in solution. This is also in agreement with dynamic LLS results, which do not show the existence of any secondary aggregates (Figure 2). We cannot 


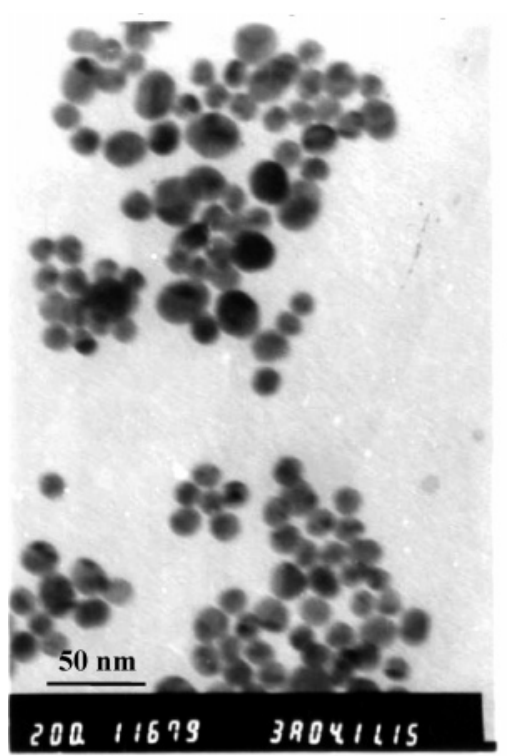

Figure 5. Typical transmission electron microscopy image of shell cross-linked core/shell hybrid gold nanoparticles.

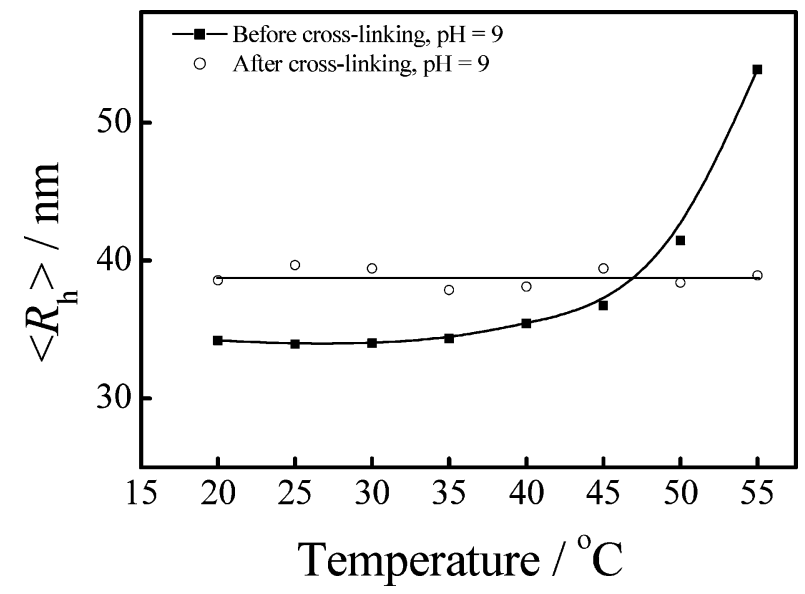

Figure 6. Variation of average hydrodynamic radius, $\left\langle R_{\mathrm{h}}\right\rangle$, as a function of temperature for core/shell gold nanoparticles before and after shell cross-linking.

observe the diblock copolymer monolayer under TEM observation. For PNIPAM and PS monolayer protected gold nanoparticles reported by Zhu et al. ${ }^{18}$ and Corbierre et al., ${ }^{19}$ they also do not observe the polymer layer by TEM. This could be due to the large difference in contrast between the gold core and the polymer layer; moreover, the monolayer may be too thin in the dry state to be clearly discerned under TEM observation. The size is in reasonable agreement with the dynamic LLS results for citrate-capped gold nanoparticles if polydispersity effects are taken into account.

Stimuli Responsiveness of Core/Shell Gold Nanoparticles. At room temperature, PDMA homopolymer is water soluble over a broad $\mathrm{pH}$ range. However, it exhibits lower critical solution temperature (LCST) phase behavior and precipitates from neutral or basic aqueous solution between 32 and $50{ }^{\circ} \mathrm{C}$, depending on its molecular weight. ${ }^{61-64}$ Considering that PDMA homopolymer is a weak polybase with $\mathrm{p} K_{\mathrm{a}} \sim 7$ and no LCST phase behavior could be observed below $\mathrm{pH} 7$, the solution $\mathrm{pH}$ was kept at 9. Figure 6 shows the temperature dependence of $\left\langle R_{\mathrm{h}}\right\rangle$ of core/shell gold nanoparticles before and after crosslinking, where the diblock copolymer concentration is about 1 $\times 10^{-4} \mathrm{~g} / \mathrm{mL}$. Each data point was obtained after the measured values were stable.

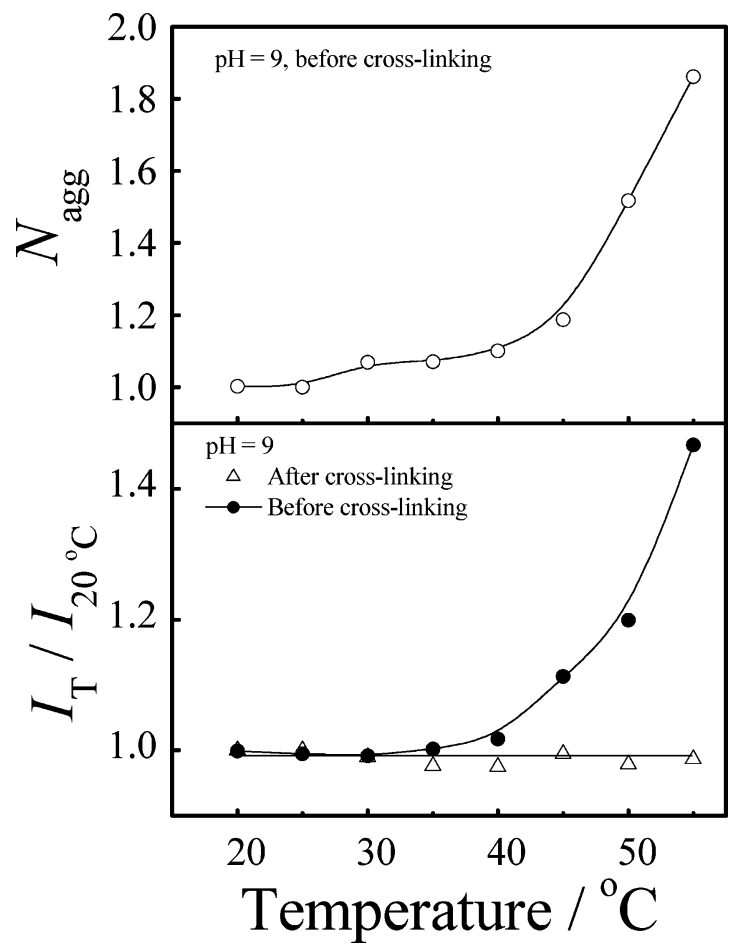

Figure 7. Temperature dependences of average aggregation number $\left(N_{\text {agg }}\right)$ of gold nanoparticles per aggregate and relative scattering light intensities (at a scattering angle of $90^{\circ}$ ) to that at $20^{\circ} \mathrm{C}$.

For the non-cross-linked core/shell hybrid gold nanoparticles, $\left\langle R_{\mathrm{h}}\right\rangle$ remains constant at $33 \mathrm{~nm}$ below $35^{\circ} \mathrm{C}$. Above $35^{\circ} \mathrm{C}$, the micellar size starts to increase, and $\left\langle R_{\mathrm{h}}\right\rangle$ increases from 33 to $54 \mathrm{~nm}$ in the temperature range $20-55^{\circ} \mathrm{C}$. The polydispersity index of the size distribution $\left(\mu_{2} / \Gamma^{2}\right)$ of non-cross-linked hybrid gold nanoparticles increases from 0.13 at $20{ }^{\circ} \mathrm{C}$ to 0.22 at 55 ${ }^{\circ} \mathrm{C}$. This indicates partial aggregation of non-cross-linked hybrid nanoparticles upon heating through the LCST of PDMA block. Figure 7 shows the temperature dependence of the average aggregation number $\left(N_{\mathrm{agg}}\right)$ of gold nanoparticles per aggregate and the relative scattered light intensity for the non-cross-linked hybrid nanoparticles at $\mathrm{pH} 9$. Here, $N_{\text {agg }}$ was calculated from the ratio of the apparent weight-average molar masses of aggregates to that of individual nanoparticles at $20^{\circ} \mathrm{C}$, assuming there is no aggregation at $20^{\circ} \mathrm{C}$. Both $N_{\text {agg }}$ and relative scattering intensity show considerable increases above $35^{\circ} \mathrm{C} . N_{\text {agg }}$ is close to 2 at $55^{\circ} \mathrm{C}$. Therefore, only weak aggregation was observed above the LCST of PDMA block; this should be due to the protection of PEO block at the outer corona, which prevents gross aggregation between hybrid gold nanoparticles.

The temperature stability of the non-cross-linked core/shell gold nanoparticles is also worth considering. Upon heating through the LCST of PDMA inner shell, the solvency was tuned to desolvate the PDMA block. Some internal reorganization should occur, leading to hybrid nanoparticles with cores comprising both the gold core and PDMA block, stabilized by the coronal PEO chains. Higher aggregation numbers might be expected for these new hybrid nanoparticles, since the hydrophilic/hydrophobic balance has changed significantly. The surface area per grafted PEO chain decreases as the aggregation proceeds until the nanoparticles are stabilized by a sufficient number of PEO chains at the surface of the aggregates.

It is interesting to note that, for the shell cross-linked hybrid core/shell gold nanoparticles, heating their aqueous solution at a concentration of $1 \times 10^{-4} \mathrm{~g} / \mathrm{mL}$ results in absolutely no changes in $\left\langle R_{\mathrm{h}}\right\rangle$ in the temperature range $20-55{ }^{\circ} \mathrm{C}$. This 


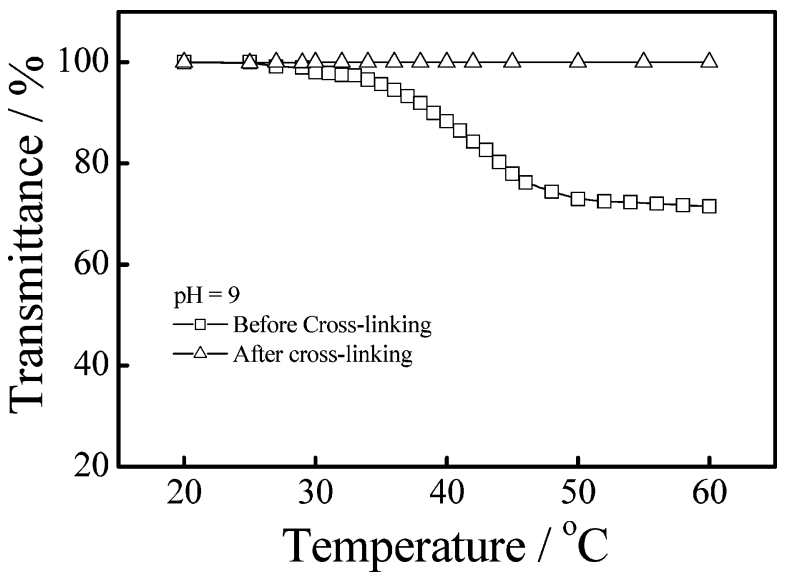

Figure 8. Temperature dependence of the transmittance of core/shell gold nanoparticles before and after shell cross-linking. The detecting wavelength is at $800 \mathrm{~nm}$.

indicates that shell cross-linking of the PDMA inner layer has led to permanent core/shell hybrid nanostructures. This is consistent with the covalent stabilization via quaternization with a bifunctional agent. ${ }^{42}$ The cross-linking has a 2-fold effect on the hybrid nanoparticles: first, quaternization suppresses the LCST phase behavior of PDMA block and renders them permanently hydrophilic; second, cross-linking results in a covalently connected network surrounding the gold nanoparticles and there is no chance for the structural arrangement of diblock copolymer chains between different hybrid nanoparticles. Overall, the shell cross-linked hybrid core/shell gold nanoparticles exhibit excellent colloidal stability at elevated temperatures compared to that before cross-linking.

Figure 8 shows the temperature dependences of the transmittances of core/shell hybrid gold nanoparticles at a concentration of about $8 \times 10^{-3} \mathrm{~g} / \mathrm{mL}$ before and after shell cross-linking, where the detecting wavelength is $800 \mathrm{~nm}$ to avoid interference of surface plasmon band. For the non-cross-linked core/shell gold nanoparticles, it can be clearly seen that gold nanoparticles coated with PEO- $b$-PDMA-SH monolayer exhibit thermoresponsive aggregation at high temperatures and the solution turns slightly opaque. Above $50^{\circ} \mathrm{C}$, PDMA is becoming completely hydrophobic, the aggregation between nanoparticles ceases, and the transmittance stabilizes at about $70 \%$. This is due to the steric hindrance of soluble PEO chains at the outer corona. Zhu et al. has reported the thermoresponsive aggregation of PNIPAM monolayer protected gold nanoparticles: the aggregation cannot be prevented and the solution becomes completely opaque with transmittance $<1 \% .{ }^{18}$ Visual inspection of non-cross-linked hybrid gold nanoparticle solution tells us that the heatinginduced aggregation is a reversible process; i.e., cooling the nanoparticle solution from $55^{\circ} \mathrm{C}$ to room temperature can reverse the slightly opaque solution back to the initially clear one. Figure 8 also reveals that, for the shell cross-linked hybrid gold nanoparticles, transmittance remains constant at about $100 \%$ in the whole temperature range $20-60{ }^{\circ} \mathrm{C}$. This again confirms that shell cross-linking has successfully fixed the threelayer hybrid core/shell nanostructure.

It should be noted that PDMA is a weak polybase with $\mathrm{p} K_{\mathrm{a}}$ $\sim 7$ 7 $^{65}$ thus the PEO- $b$-PDMA monolayer coated gold nanoparticles should also be $\mathrm{pH}$-responsive. Figure 9 shows the $\mathrm{pH}$ dependence of $\left\langle R_{\mathrm{h}}\right\rangle$ for core/shell gold nanoparticles before and after shell cross-linking. At $\mathrm{pH}<7$, the PDMA blocks are cationic due to protonation. As a result, the inner PDMA shell should expand due to charge expulsion between neighboring DMA units, and $\left\langle R_{\mathrm{h}}\right\rangle$ of the non-cross-linked nanoparticles

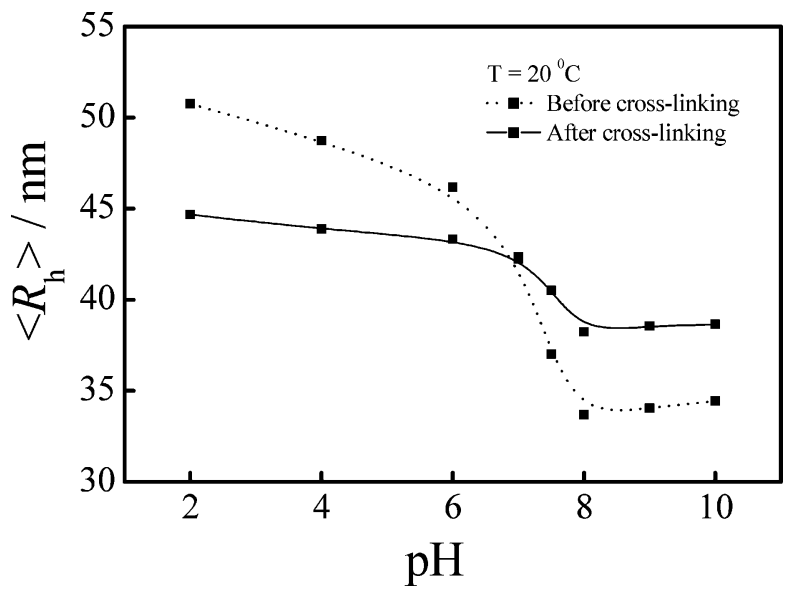

Figure 9. $\mathrm{pH}$ dependence of average hydrodynamic radius $\left\langle R_{\mathrm{h}}\right\rangle$ of core/ shell gold nanoparticles before and after shell cross-linking at $20^{\circ} \mathrm{C}$.

increases from $33 \mathrm{~nm}$ at $\mathrm{pH} 9$ to $51 \mathrm{~nm}$ at $\mathrm{pH} 2$. Most of the size change takes place in the $\mathrm{pH}$ range $6-8$, which is consistent with the $\mathrm{p} K_{\mathrm{a}}$ known for PDMA homopolymers. $\left\langle R_{\mathrm{h}}\right\rangle$ of the shell cross-linked nanoparticles also has $\mathrm{pH}$ dependence: it increases from $38 \mathrm{~nm}$ at $\mathrm{pH} 9$ to $45 \mathrm{~nm}$ at $\mathrm{pH}$ 2. The $\mathrm{pH}$ dependence of hybrid nanoparticle size after cross-linking is less prominent than that before cross-linking. Cross-linking of PDMA inner shell results in the formation of a polymer network, and this may restrict its swelling. It is also reasonable if we consider that part of the DMA units has already been permanently quaternized during cross-linking, then the nanoparticle size exhibits less $\mathrm{pH}$ dependence.

\section{Conclusion}

Citrate-capped gold nanoparticles are coated with a monolayer composed of a double hydrophilic block copolymer, namely, PEO- $b$-PDMA-SH. The resulting hybrid nanoparticles take a three-layer microstructure with a gold core, PDMA inner shell, and PEO outer corona. The stimuli responsiveness of core/shell gold nanoparticles before and after shell cross-linking is studied in detail. At $\mathrm{pH} 9$, the average hydrodynamic radius, $\left\langle R_{\mathrm{h}}\right\rangle$, of non-cross-linked hybrid gold nanoparticles starts to increase above $35{ }^{\circ} \mathrm{C}$ due to the lower critical solution temperature (LCST) behavior of the PDMA block in the inner shell. In contrast, $\left\langle R_{\mathrm{h}}\right\rangle$ of the shell cross-linked hybrid gold nanoparticles is independent of temperature. Core/shell gold nanoparticles before and after shell cross-linking both exhibit reversible swelling behavior upon varying the solution $\mathrm{pH}$. Compared to the non-cross-linked core/shell gold nanoparticles, the shell cross-linking of the hybrid gold nanoparticles leads to a permanent core/shell nanostructure with much higher colloidal stability.

Acknowledgment. This work is supported by an Outstanding Youth Fund (50425310) from the National Natural Scientific Foundation of China (NNSFC) and the "Bai Ren" Project of the Chinese Academy of Sciences.

\section{References and Notes}

(1) Daniel, M. C. Astruc, D. Chem. Rev. 2004, 104, 293.

(2) Shan, J.; Nuopponen, M.; Jiang, H.; Viitala, T.; Kauppinen, E.; Kontturi, K.: Tenhu, H. Macromolecules 2005, 38, 2918.

(3) Shiway, A. N.; Katz, E.; Willner, I. ChemPhysChem 2000, 1, 18.

(4) Remacle, F.; Levine, R. D. ChemPhysChem 2001, 2, 20.

(5) Templeton, A. C.; Wuelfing, W. P.; Murray, R. W. Acc. Chem. Res. 2000, 33, 27.

(6) Brust, M.; Walker, M.; Bethell, D.; Schiffrin, D. J.; Whyman, R. J. Chem. Soc., Chem. Commun. 1994, 801. 
(7) Brust, M.; Fink, J.; Bethell, D.; Schiffrin, D. J.; Kiely, C. J. Chem. Soc., Chem. Commun. 1995, 1655.

(8) Nuss, S.; Böttcher, H.; Wurm, H.; Hallensleben, M. L. Angew. Chem., Int. Ed. 2001, 40, 4016.

(9) Ohno, K.; Koh, K.-M.; Tsujii, Y.; Fukuda, T. Macromolecules 2002, 35,8989 .

(10) Mandal, T. K.; Fleming, M. S.; Walt, D. R. Nano Lett. 2002, 2, 3.

(11) Raula, J.; Shan, J.; Nuopponen, M.; Niskanen, A.; Jiang, H.; Kauppinen, E.; Tenhu, H. Langmuir 2003, 19, 3499.

(12) Kaholek, M.; Lee, W.-K.; Ahn, S.-J.; Ma, H.; Caster, K. C.; LaMattina, B.; Zauscher, S. Chem. Mater. 2004, 16, 3688.

(13) Wuelfing, W. P.; Gross, S. M.; Miles, D. T.; Murray, R. W. J. Am. Chem. Soc. 1998, 120, 12696.

(14) Corbierre, M. K.; Cameron, N. S.; Sutton, M.; Mochrie, S. G. J.; Lurio, L. B.; Ruhm, A.; Lennox, R. B. J. Am. Chem. Soc. 2001, 123, 10411

(15) Mangeney, C.; Ferrage, F.; Aujard, I.; Marchi-Artznern, V.; Jullien, L.; Ouari, O.; Rekai, E. D.; Laschewsky, A.; Vikholm, I.; Sadowski, J. W. J. Am. Chem. Soc. 2002, 124, 5811.

(16) Lowe, A. B.; Sumerlin, B. S.; Donovan, M. S.; McCormick, C. L. J. Am. Chem. Soc. 2002, 124, 11562.

(17) Shan, J.; Nuopponen, M.; Jiang, H.; Kauppinen, E.; Tenhu, H. Macromolecules 2003, 36, 4526.

(18) Zhu, M.; Wang, L.; Exarhos, G. J.; Li, A. D. Q. J. Am. Chem. Soc. 2004, 126, 2656.

(19) Corbierre, M. K.; Cameron, N. S.; Lennox, R. B. Langmuir 2004, $20,2867$.

(20) Chiefari, J.; Chong, Y. K.; Ercole, F.; Krstina, J.; Jeffery, J.; Le, T. P. T.; Mayadunne, R. T. A.; Meijs, G. F.; Moad, C. L.; Moad, G.; Rizzardo, E.; Thang, S. H. Macromolecules 1998, 31, 5559.

(21) Chong, Y. K.; Krstina, J.; Le, T. P. T.; Moad, G.; Postma, A.; Rizzardo, E.; Thang, S. H. Macromolecules 2003, 36, 2256.

(22) Shan, J.; Nuopponen, M.; Jiang, H.; Kauppinen, E.; Tenhu, H. Macromolecules 2003, 36, 4526 . 4671.

(23) Shan, J.; Chen, J.; Nuopponen, M.; Tenhu, H. Langmuir 2004, 20,

(24) Zhang, L.; Eisenberg, A. J. Am. Chem. Soc. 1996, 118, 3168

(25) Chong, Y. K.; Le, T. P. T.; Moad, G.; Rizzardo, E.; Thang, S. H. Macromolecules 1999, 32, 2071.

(26) Luo, L.; Eisenberg, A. Langmuir 2001, 17, 6804.

(27) Nuopponen, M.; Ojala, J.; Tenhu, H. Polymer 2004, 45, 3643.

(28) Virtanen, J.; Tenhu, H. Macromolecules 2000, 33, 5970.

(29) Arotcarena, M.; Heise, B.; Ishaya, S.; Laschewsky, A. J. Am. Chem. Soc. 2002, 124, 3787.

(30) Chung, J. E.; Yokoyama, M.; Okano, T. J. Controlled Release 2000, 65,93

(31) Virtanen, J.; Arotcarena, M.; Heise, B.; Ishaya, S.; Laschewsky,

A.; Tenhu, H. Langmuir 2002, 18, 5360.

(32) Thurmond, K. B.; Kowalewski, T.; Wooley, K. L. J. Am. Chem. Soc. 1996, 118, 7239 .

(33) Huang, H.; Kowalewski, T.; Remsen, E. E.; Gertzmann, R.; Wooley, K. L. J. Am. Chem. Soc. 1997, 119, 11653.

(34) Bütün, V.; Billingham, N. C.; Armes, S. P. J. Am. Chem. Soc. 1998 120,12135 . 1415.
(36) Becker, M. L.; Remsen, E. E.; Wooley, K. L. J. Polym. Sci., Part A: Polym. Chem. 2001, 39, 4152.

(37) Sanji, T.; Nakatsuka, Y.; Ohnishi, S.; Sakurai, H. Macromolecules 2000, 33, 8524 .

(38) Underhill, R. S.; Liu, G. Chem. Mater. 2000, 12, 2082.

(39) Weaver, J. V. M.; Tang, Y.; Liu, S.; Iddon, P. D.; Grigg, R.; Billingham, N. C.; Armes, S. P.; Hunter, R.; Rannard, S. P. Angew. Chem., Int. Ed. 2004, 43, 1389.

(40) Liu, S.; Armes, S. P. J. Am. Chem. Soc. 2001, 123, 9910.

(41) Liu, S.; Ma, Y.; Armes, S. P.; Perruchot, C.; Watts, J. F. Langmuir 2002, 18, 7780

(42) Liu, S.; Weaver, J. V. M.; Tang, Y.; Billingham, N. C.; Armes, S.

P.; Tribe, K. Macromolecules 2002, 35, 6121.

(43) Liu, S.; Weaver, J. V. M.; Save, M.; Armes, S. P. Langmuir 2002, 18,8350 .

(44) Pilon, L. N.; Armes, S. P.; Findlay, P.; Rannard, S. P. Langmuir 2005, 21, 3808

(45) Fujii, S.; Cai, Y.; Weaver, J. V. M.; Armes, S. P. J. Am. Chem. Soc. 2005, 127, 7304

(46) Joralemon, M. J.; Murthy, K. S.; Remsen, E. E.; Becker, M. L.;

Wooley, K. L. Biomacromolecules 2004, 5, 903.

(47) Yan, X. H.; Liu, F. T.; Li, Z.; Liu, G. J. Macromolecules 2001, $34,9112$.

(48) (a) Kang, Y.; Taton, T. A. Angew. Chem., Int. Ed. 2005, 44, 409.

(b) Kang, Y.; Taton, T. A. J. Am. Chem. Soc. 2003, 125, 5650.

(49) Cölfen, H. Macromol. Rapid Commun. 2001, 22, 219.

(50) Gohy, J. F.; Lohmeijer, B. G. G.; Varshney, S. K. Macromolecules 2002, 35, 9748 .

(51) Hadjikallis, G.; Hadiyannakou, S. C.; Vamvakaki, M. Polymer 2002 43,7269 .

(52) Rodriguez-Hernandez, J.; Lecommandoux, S. J. Am. Chem. Soc 2005, 127, 2026.

(53) Arotcarena, M.; Heise, B.; Ishaya, S.; Laschewsky, A. J. Am. Chem Soc. 2002, 124, 3787.

(54) Andre, X.; Zhang, M. F.; Muller, A. H. E. Macromol. Rapid Commun. 2005, 26, 558

(55) Mitsukami, Y.; Donovan, M. S.; Lowe, A. B.; McCormick, C. L. Macromolecules 2001, 34, 2248.

(56) Grabar, K. C.; Freeman, R. G.; Hommer, M. B.; Natan, M. J. Anal. Chem. 1995, 67, 735.

(57) Chong, B. Y. K.; Le, T. P. T.; Moad, G.; Rizzardo, E.; Thang, S. H. Macromolecules 1999, 32, 2071.

(58) Kaholek, M.; Lee, W.-K.; Ahn, S.-J.; Ma, H.; Caster, K. C. LaMattina, B.; Zauscher, S. Chem. Mater. 2004, 16, 3688.

(59) Templeton, A. C.; Pietron, J. J.; Murray, R. W.; Mulvaney, P. J. Phys. Chem. B 2000, 104, 564

(60) Lu, Y.; Yin, Y.; Li, Z.-Y.; Xia, Y. Nano Lett. 2002, 2, 785.

(61) Butun, V.; Billingham, N. C.; Armes, S. P. Chem. Commun. 1997, 671.

(62) Lee, A. S.; Gast, A. P.; Butun, V.; Armes, S. P. Macromolecules 1999, 32,4302 .

(63) Butun, V.; Armes, S. P.; Billingham, N. C. Polymer 2001, 42, 5993.

(64) Vamvakaki, M.; Billingham, N. C.; Armes, S. P. Macromolecules 1999, 32, 2088

(65) Adam, M.; Delasanti, M. Macromolecules 1985, 18, 1760. 